\title{
Qingkailing injection ameliorates cerebral ischemia-reperfusion injury and modulates the AMPK/NLRP3 Inflammasome Signalling pathway
}

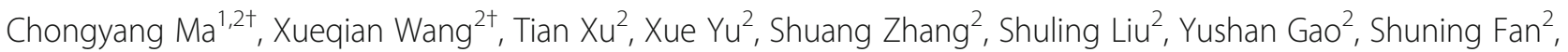
Changxiang Li ${ }^{2}$, Changming Zhai ${ }^{2}$, Fafeng Cheng ${ }^{2^{*}}$ and Qingguo Wang ${ }^{2^{*}}$

\begin{abstract}
Background: Cerebral ischemia is the second-leading cause of death and the main cause of permanent adult disabilities worldwide. Qingkailing (QKL) injection, a patented Chinese medicine approved by the China Food and Drug Administration, has been widely used in clinical practice to treat cerebral ischemia in China. The NOD-like receptor pyrin 3 (NLRP3) inflammasome is activated in cerebral ischemia and thus, is an effective therapeutic target. AMP-activated protein kinase (AMPK) is an important regulator inhibiting NLRP3 inflammasome activation.

Methods: We investigated the potential of QKL injection to provide neuroprotection after cerebral ischemia in a rat model of middle cerebral artery occlusion (MCAO). Adult male Sprague-Dawley rats (210-230 g) were randomly divided into three groups which consist of sham, MCAO and $3 \mathrm{ml} / \mathrm{kg}$ QKL. Rats in the QKL group received intraperitoneal injections of $3 \mathrm{ml} / \mathrm{kg} \mathrm{QKL}$, while rats in other groups were given saline in the same volumes. After $90 \mathrm{~min}$ ischemia and $24 \mathrm{~h}$ reperfusion, neurological function, laser speckle imaging, brain infarction, brain water content and brain blood barrier permeability were examined and cell apoptosis at prefrontal cortex were evaluated $24 \mathrm{~h}$ after MCAO, and western blot and real-time quantitative polymerase chain reaction was also researched, respectively.

Results: Intraperitoneal administration of QKL alleviated neurological deficiencies, cerebral infarction, blood-brain barrier permeability, brain oedema and brain cell apoptosis after MCAO induction. QKL decreased pro-inflammatory cytokines, TNF-a, IL-6 and IL-1 $\beta$, and increased anti-inflammatory cytokines, IL-4 and IL-10. Furthermore, QKL activated phosphorylated AMPK, decreased oxidative stress and decreased NLRP3 inflammasome activation.

Conclusions: QKL relieved cerebral ischemia reperfusion injury and suppressed the inflammatory response by inhibiting AMPK-mediated activation of the NLRP3 inflammasome. These results suggest that QKL might have potential in treating brain inflammatory response and attenuating the cerebral ischemia-reperfusion injury.
\end{abstract}

Keywords: Cerebral ischemia-reperfusion injury, NLRP3 inflammasome, Chinese medicine, Qingkailing injection

* Correspondence: fafengcheng@gmail.com; wangqg8558@163.com

${ }^{\dagger}$ Chongyang $\mathrm{Ma}$ and Xueqian Wang contributed equally to this work.

${ }^{2}$ School of Traditional Chinese Medicine Department, Beijing University of

Chinese Medicine, 11 Beisanhuandong Road, Chao Yang District, Beijing

100029, China

Full list of author information is available at the end of the article

(c) The Author(s). 2019 Open Access This article is distributed under the terms of the Creative Commons Attribution 4.0 International License (http://creativecommons.org/licenses/by/4.0/) which permits unrestricted use, distribution, and reproduction in any medium, provided you give appropriate credit to the original author(s) and the source, provide a link to the Creative Commons license, and indicate if changes were made. The Creative Commons Public Domain Dedication waiver (http://creativecommons.org/publicdomain/zero/1.0/) applies to the data made available in this article, unless otherwise stated. 


\section{Background}

Cerebral Ischemia is the second-leading cause of death behind ischemic heart disease, and is the main cause of permanent adult disabilities worldwide $[1,2]$. Thrombolytic therapy is the only therapy recommended to treat cerebral ischemia, however, it is limited by a very narrow therapeutic window and a high risk of haemorrhagic complications [3]. Therefore, a focus on understanding the detailed pathological process behind cerebral ischemia may facilitate the creation of more novel and efficient therapeutic agents. The importance of innate immune mechanisms as a response to cerebral ischemiareperfusion injury has been recognized recently [4]. Following a transient blockage of cerebral blood flow, dangerous molecular signals are released from dead or dying cells [5]. These signals, known as damage-associated molecular patterns (DAMPs) and pathogen-associated molecular patterns (PAMPs), stimulate the initial activation of innate immune responses during the progression of cerebral ischemia via extracellular and intracellular pattern recognition receptors (PRRs). Inflammasomes are activated by some PRR signals, induce maturation and secretion of some inflammatory cytokines, and initiate cell pyroptosis, a form of programmed inflammatory cell death [6].

Recent research has highlighted a novel inflammasome, the nucleotide-binding oligomerization domain (NOD)-like receptor (NLR) Pyrin domain containing 3 (NLRP3) inflammasome that acts as a key regulator in detecting cellular damage and modulating inflammatory responses to aseptic tissue injury post-stroke [7]. NLRP3 inflammasome is one of the best characterized inflammasomes to date, and is the most strongly relevant in cerebral ischemia. The NLRP3 inflammasome comprises three kinds of cytoplasmic proteins: 1) NLRP3, 2) apoptosis-associated speck-like protein containing a CARD (ASC), and 3) a precursor of caspase-1, which cleaved formation leads to maturation and secretion of IL-1 $\beta$ and IL18, and induce cellular pyroptosis [8]. In the central nervous system (CNS), an NLRP3 inflammasome signalling pathway was activated and the expression of core proteins, such as NLRP3, ASC, caspase-1, IL-1, and IL-18, were upregulated in vitro and in vivo under ischemic conditions [9]. Suppressing the NLRP3 inflammasome activation was also proven to be associated with better functional outcomes, decreased infarction volumes and oedema formation, preserved blood brain barrier (BBB) permeability, and reduced inflammatory pathology in a transient middle cerebral artery occlusion (tMCAO) rat model $[10,11]$.

AMP-activated protein kinase (AMPK) is a master sensor of cellular energy balance and a fundamental regulator of cellular carbohydrate and fat metabolism and ATP conservation and synthesis. An increased
AMP: ATP ratio leads to AMPK kinase activation and then activates AMPK to switch off ATP-consuming pathways and switch on ATP-generating pathways [12]. Recently, AMPK was found to play a role in regulating NLRP3 inflammasome activation. According to a newly published review article, activating AMPK signals leads to inhibition of the NLRP3 inflammasome via improved autophagy, alleviation of ER stress, activation of SIRT1, and regulation of mitochondrial homeostasis [13]. AMPK activation represents a potential protective mechanism in the early stages of cerebral ischemia [14]. Therefore, the AMPK/NLRP3 inflammasome pathway has the potential to be a therapeutic target in the treatment of cerebral ischemia.

Qingkailing (QKL) injection, a patented Chinese medicine that is approved by the China Food and Drug Administration to treat cerebral ischemia (registration information can be accessed here: http://samr.cfda.gov. cn/WS01/CL0412/), was originally prepared by a group of scientists at the Beijing University of Chinese Medicine in the 1970s. The substance was formulated by modifying a famous traditional Chinese medicine, Angongniuhuang [15], which were pills composed of Radix Isatidis, Flos Lonicerae, Concha Margaritifera Usta, baicalin, Fructus gardeniae, cholic acid, hyodeoxycholic acid, and Cornu Bubaliand. Angongniuhuang pills had been widely used in clinical practice to treat cerebral ischemia for over 30 years. A recently updated systematic review and meta-analysis published by our group evaluated 18 randomized controlled trials (RCTs, 1722 patients). The results indicated that QKL Injection combined with a conventional therapy improved the efficiency of treatment for cerebral ischemia compared to conventional treatment alone [16, 17]. The specific mechanism behind the biological effect of QKL at that time, however, was not fully understood. Recent real world study showed that QKL decreased the level of Creactive protein, white blood cells and other abnormal inflammatory indicators clinically, highlighting a pharmacological effect on immune system [18]. And our unpublished systems pharmacology results showed that QKL may regulate post-stroke inflammatory response and modulate pattern recognition receptors signal. In vitro evidence showed that QKL significantly supressed the activation of microglia and modulated secretion of inflammatory factors, including TNF- $\alpha, \mathrm{COX}-2$ and iNOS [19].

In the present study, we hypothesized that QKL could ameliorate cerebral ischemia-reperfusion injury and modulate the AMPK/NLRP3 inflammasome signalling pathway. To test our hypothesis, we designed an experiment that could quantify the brain infarct volume and blood-brain barrier permeability and detect the level of activation of the AMPK/NLRP3 pathway. 


\section{Methods}

\section{Ethical statement}

The animal experimental design and protocols used in this study were approved by the Ethics Review Committee for Animal Experimentation at the Beijing University of Chinese Medicine. All the experimental procedures were performed in accordance with the Regulations for the Administration of Affairs Concerning Experimental Animals approved by the State Council of People's Republic of China.

\section{Drugs and reagents}

Qingkailing (QKL) was purchased from Shenwei Pharmaceutical Co., Ltd. (No. Z13020935, China). The protease inhibitor, radioimmunoprecipitation assay (RIPA) lysis buffer, and enhanced chemiluminescence (ECL) reagent were obtained from Vazyme Biotech (Nanjing, China). The antibodies against GAPDH, pAMPK, and AMPK were purchased from Cell Signaling Technology (Danvers, MA USA), and the antibodies against NLRP3, ASC, IL-1 $\beta$ and caspase-1 were obtained from Abcam (Cambridge, UK).

\section{Animals}

Adult male Sprague-Dawley rats (210-230 g) were provided by the Vital River Laboratory Animal Technology (number SCXK 2016-0006) (Beijing, China). The rats were housed in the experimental animal centre of Beijing University of Chinese Medicine, which was maintained at $25^{\circ} \mathrm{C} \pm 1{ }^{\circ} \mathrm{C}$ with $65 \% \pm 5 \%$ humidity on a 12 -h light/dark cycle for at least 1 week before the experiments. Animals were given food and water freely.

\section{Model establishment of tMCAO and treatment}

All animals were fasted overnight but allowed free access to water and were assigned to three groups according to the random number table ( $n=22$ rats each group) as follows: (1) Sham group, (2) MCAO group and (3) QKL group. The transient MCAO model was performed as described previously [20]. Briefly, rats were anesthetized $4 \%$ isoflurane until no corneal reflex and toe-pinching were observed. And 1\% isoflurane in a mixture of $30 \%$ oxygen and $70 \%$ nitrous oxide was used during tMCAO surgery. A poly-L-lysine-coated nylon suture was inserted from the right external carotid artery into the common carotid artery to occlude the middle cerebral artery. Rats in the sham group were suffered similar operation without inserting sutures. Cerebral reperfusion was carried out by carefully removing the suture after $90 \mathrm{~min}$ from the onset of occlusion. The body temperature was controlled at $37^{\circ} \mathrm{C}$ by an electric blanket. Rats in the QKL group received intraperitoneal injections of QKL dissolved in saline at a dose of $3 \mathrm{ml} / \mathrm{kg}$. Rats in the MCAO group and Sham group received intraperitoneal injections of saline in the same volumes. The first injection was performed immediately after model establishment, followed by an administration after $4 \mathrm{~h}$, and then once every $12 \mathrm{~h}$ thereafter. At $24 \mathrm{~h}$ after reperfusion, euthanasia was performed by excessive inhalation of isoflurane. Death was monitored by the cardiac activity and respiration. All efforts were made to minimize animal suffering and to reduce the Number of animals used. All efforts were made to minimize animal suffering and to reduce the Number of animals used. After sacrificed, all the brains were taken out quickly for TTC staining ( $n=10$ each group), assessment of BBB permeability and brain water containing $(n=6$ each group), histomorphological assays ( $n=3$ each group) or protein detection ( $\mathrm{n}=3$ each group).

\section{Neurological assessment}

The neurological deficit for each rat was measured $24 \mathrm{~h}$ after reperfusion by an investigator who did not know the experimental groupings using the a five-point neurological scale [21]: score $0=$ no apparent deficits; score $1=$ failure to fully extend the right forepaw; score $2=$ circling to the right; score 3 =falling or leaning over to the right; score $4=$ no spontaneous walking and a depressed level of consciousness; score 5 = dead.

\section{Cerebral blood flow measurement}

After neurological assessment, cerebral blood flow (CBF) was measured by Lasser Doppler perfusion monitor (PeriCam PSI System) as previously described [22, 23]. Rats were anesthetized with the dose of $35 \mathrm{mg} / \mathrm{kg}$ pentobarbital sodium solution $(3 \%, \mathrm{w} / \mathrm{v})$. Laser scanning imaging measurements were performed on the intact skull. Real-time CBF changes were recorded every $1 \mathrm{~min}$ with a CCD camera and a Pericam PSI System (Perimed) that was placed roughly $10 \mathrm{~cm}$ above the brain. From the measurements obtained, the relative ipsilateral: contralateral CBF ratio was calculated.

\section{Infarct volume assessment}

Following the neurological function evaluation and cerebral blood flow measurement, the rats were sacrificed as described previously and the brains were harvested for TTC staining [24, 25]. Brains were cut into serial coronal sections ( $2 \mathrm{~mm}$ thickness) and soaked in $2 \%$ TTC phosphate buffer at $37^{\circ} \mathrm{C}$ for $15 \mathrm{~min}$ in the dark place. These sections were soaked in $4 \%$ paraformaldehyde phosphate buffer for $30 \mathrm{~min}$. An electronic scanner (Tsinghua Unisplendour A688, Xi'an, China) was used to capture images of ordered brain sections. Normal brain tissues stained red while infarct tissues did not stain (white). Areas of red and white staining were measured using a computer colour multimedia image analysis system (Image-Pro Plus6.0, Media Cybernetics, Wyoming, 
USA). The percent of infarction was calculated using the following equation: \%Infarct volume = Infarct volume/ Total volume of slice $\times 100$.

\section{Brain water content}

Brain oedema was also determined $24 \mathrm{~h}$ after reperfusion. The brains were separated and sliced. The slices were immediately weighed and recorded as the wet weight, and then they were put in an oven at $100{ }^{\circ} \mathrm{C}$ for over $24 \mathrm{~h}$ to obtain the dry weight. The water content was expressed as using following formula: [(wet weight) - (dry weight)]/(wet weight) $\times 100 \%$.

\section{BBB permeability}

BBB permeability was measured using Evans Blue (EB) extravasation at $24 \mathrm{~h}$ after reperfusion [26]. In brief, EB dye $(2 \%, 4 \mathrm{~mL} / \mathrm{kg})$ was injected through caudal vein at a dose of $2 \mathrm{ml} / \mathrm{kg}$ and allowed to circulate for $1 \mathrm{~h}$. The rats were anesthetized using pentobarbital sodium solution and perfused transcardially using cold saline to remove intravascular EB dye. After sacrificed, the entire brain of each animal was removed, divided into ipsilateral and contralateral hemispheres, and homogenized in physiological phosphate buffered saline (PBS). Trichloroacetic acid was then added to precipitate the proteins, and the samples were cooled and centrifuged. The supernatant was measured for EB absorbance at $620 \mathrm{~nm}$ using a spectrophotometer. Taking the optical density of the contralateral hemisphere as background, the fold change was determined by the following formula: (ipsilateralcontralateral)/contralateral.

\section{Nissl staining}

Coronal brain sections were stained with thionine as previously described to assess ischemic/reperfusion injury [27]. Necrotic neurons were identified by the disappearance of Nissl bodies in the cytoplasm, shrunken intercellular spaces, and deep staining. The number of neuronal cells in the border of the infarct area was counted.

\section{TUNEL staining}

Cerebral ischemia-induced apoptosis was quantified by TdT-mediated dUTP Nick-End Labelling (TUNEL) assay (Roche Molecular Biochemicals, Mannheim, Germany) as previously described [28, 29]. TUNELpositive apoptotic cells in peri-ischemia region of cortex were counted in five random high-power fields per section by an investigator who did not know the experimental groupings. The apoptotic index was calculated as a ratio of the apoptotic cell number to the total cell number in each field.

\section{ELISA analysis}

The cortical tissue in the penumbra was washed in cold PBS and shredded below $4{ }^{\circ} \mathrm{C}$. Then cell lysates were centrifuged at $1000 \mathrm{~g}$ for $20 \mathrm{~min}$, and supernatant was stored at $-20{ }^{\circ} \mathrm{C}$. The concentration of TNF- $\alpha$, IL-6, IL$1 \beta$, IL- 4 and IL-10 in the cortex was assessed using ELISA, according to the manufacturer's protocol (Cloud-Clone Corp, Texas, USA). The total protein level was normalized for each sample to conduct ELISA [30].

\section{MDA and SOD measurement}

MDA was determined using a kit according to the manufacturer's directions (Nanjing Jiancheng Bioengineering Institute). In addition, SOD activity was determined using the xanthine oxidase method according to the manufacturer's directions (Nanjing Jiancheng Bioengineering Institute).

\section{Western blotting}

The cortical tissue in the penumbra was washed in cold PBS and shredded below $4{ }^{\circ} \mathrm{C}$. The total protein was extracted using RIPA lysis buffer containing protease inhibitor and phosphatase inhibitor, and the concentration was measured using a bicinchoninic acid (BCA) Protein Assay Kit (\#CW0014, CWbio, China). Proteins of $50 \mu \mathrm{g}$ were separated on sodium dodecyl Right (SDS)-polyacrylamide gels and transferred onto a polyvinylidene fluoride membrane (Millipore Corporation, Billerica, USA). The membrane was blocked with 5\% nonfat dry milk in Tris-buffered saline containing 0.05\% Tween-20 (TBST) buffer. They were then incubated with primary antibodies against NLRP3, ASC, caspase-1, IL-1 $\beta$, pAMPK, AMPK, and GAPDH (dilutions of 1:1000, 1: 1000, 1:1000, 1:1000, 1:2000, 1:2000, 1:10000) overnight at $4{ }^{\circ} \mathrm{C}$. The membranes were incubated for $1 \mathrm{~h}$ at room temperature with secondary antibodies coupled to horseradish peroxidase at a 1: 10000 dilution. The antigenantibody complexes were then tested by enhanced chemiluminescence (ECL) reagent and visualized on a C600 Western Blot Imaging System (Azure Biosystems, Dublin, USA). The protein levels of these proteins are expressed as relative integrated intensity normalized versus GAPDH.

\section{Quantitative real-time PCR quantitation}

RNA was isolated from the cortical tissue in the penumbra using Trizol Reagent (Invitrogen, Grand Island, NY, USA). The RNA concentration was measured using an ultraviolet spectrophotometer (UV-2000, Unico, Shanghai, China). Then, reverse transcription was performed from $2 \mu \mathrm{g}$ RNA on a T100 Thermal Cycler PCR machine (Bio-Rad, USA) using Revert Aid First Strand cDNA Synthesis Kit (Thermo Fisher Scientific, Waltham, WA, USA,). Quantitative PCR was performed according to 
the manufacturers' instructions using SYBR Select Master MIX (K1622, Applied Biosystems, CA, USA) and a Real-Time PCR machine (Bio-Rad, USA). Expression values of the targeted genes were normalized to the corresponding expression of $\beta$-actin. The $2^{-\Delta \Delta C t}$ method was used to calculate the relative gene expression. Sequence-specific primers are listed in Table 1.

\section{Statistical analysis}

All experiments were performed in triplicate and the data are expressed as means \pm standard error measurements (SEM). One-way analysis of variance (ANOVA) were employed for the statistical analysis using SPSS 22.0 software (SPSS, Inc.). $P<0.05$ was defined as significant difference.

\section{Results}

Effects of QKL on regional cerebral blood fluid, neurological score, and infarct volume

To evaluate the hemodynamic effects induced by the QKL injection, we analysed regional CBF in the ipsilateral and contralateral tissues using the PeriCam PSI System (PerimedAB, Sweden). We used the ipsilateral: contralateral $\mathrm{rCBF}$ ratio to evaluate the effect of $\mathrm{QKL}$ on the decrease in blood flow post-stroke $(\mathrm{F}(2,27)=12.73$, $p=0.0003)$. As shown in Fig. 1a-b, $24 \mathrm{~h}$ after the cerebral ischemia-reperfusion injury, the rCBF of the ipsilateral side, after being normalized by the contralateral side, decreased to $69.92 \%$ (Vs Sham, $p=0.0002$ ) and QKL reversed the data to $83.85 \%$ (Vs MCAO, $p=0.0181$ ). We also observed that QKL improved neurological function and induced brain infarct volume as shown in Fig. 1c-e.

\section{Effects of QKL on BBB permeability and brain water content}

We evaluated the BBB permeability by assessing Evans blue $(\mathrm{EB})$ extravasation at $24 \mathrm{~h}$ post-stroke $(\mathrm{F}(2,15)=$ $6.575, p=0.0023)$. The EB leakage was significantly reduced in the QKL group than in the MCAO group as shown in Fig. 2a-b (Vs MCAO, $p=0.0065$ ). And the cerebral ischemia-reperfusion injury resulting $\mathrm{BBB}$ leakage caused an increase in brain water content (Vs Sham, $p=0.0024)$. Although there is a significant difference among these groups $(\mathrm{F}(2,15)=2.166, p=0.0015)$, as shown in Fig. 2c, QKL administration led to a decrease in brain oedema in the ipsilateral hemisphere, and the difference was not significant (Vs MCAO, $p>0.05$ ).

\section{Effects of QKL on post-stroke cell survival}

Cell damage after cerebral ischemia-reperfusion injury was estimated using Nissl staining. As shown in Fig. 3ab, most cells shrank in size, although the intercellular space was increased and had deep colour staining in the MCAO group. However, these characteristic changes were improved after QKL treatment. Furthermore, more intact cells were present in the penumbra of the ischemic cortex in the QKL-treated rodents than in the MCAO group (Vs MCAO, $p=0.0003$ ). Similarly, terminal deoxynucleotidyl transferase dUTP nick end labelling (TUNEL) positive cells were examined in the cortex after MCAO. As shown in Fig. 3c-d, QKL reduced the percentage of apoptotic cells significantly (Vs MCAO, $p=0.0006)$.

\section{Effects of QKL on upregulation of inflammatory cytokines by cerebral ischemia}

Enzyme linked immunosorbent assay (ELISA) analysis was used to evaluate the amount of pro-inflammatory (TNF- $\alpha$, IL-1 $\beta$ and IL-6) and anti-inflammatory cytokines (IL-4 and IL-10). As shown in Fig. 4a, QKL treatment decreased TNF $\alpha$ significantly (Vs MCAO, $p=0.0001$ ), after TNF- $\alpha$ had been upregulated by the cerebral ischemia-reperfusion injury. A similar phenomenon was observed when detecting IL- 6 and IL$1 \beta$ (Vs MCAO, $p=0.02, p=0.0227$, respectively), which is shown in Fig. 4b-c. The anti-inflammatory factors were upregulated in the QKL-treatment group compared to the MCAO group. QKL significantly increased the level of IL-4 (Vs MCAO, $p=0.0419$ ) and increased the level of IL-10, but this difference was not significant (Vs $\mathrm{MCAO}, p>0.05)$.

\section{Effects of QKL on the AMPK/NLRP3 inflammasome signalling pathway}

To understand the anti-stroke mechanism of QKL, we determined the protein expression levels of NLRP3, ASC, pro-caspase-1, cleaved-caspase-1, pro-IL-1 $\beta$ and cleaved-IL-1 $\beta$ in the brain tissue of rats (Fig. 5a). As

Table 1 Real-time fluorescent quantitative PCR primers

\begin{tabular}{lll}
\hline Gene names & Forward & Reverse \\
\hline NLRP3 & CAGCGATCAACAGGCGAGAC & AGAGATATCCCAGCAAACCTATCCA \\
ASC & GCTGAGCAGCTGCAAACGA & ACTTCTGTGACCCTGGCAATGA \\
Caspase-1 & ACTCGTACACGTCTTGCCCTCA & CTGGGCAGGCAGCAAATTC \\
IL-1 $\beta$ & CCCTGAACTCAACTGTGAAATAGCA & CCCAAGTCAAGGGCTTGGAA \\
GAPDH & GAACATCATCCCTGCATCCA & CCAGTGAGCTTCCCGTTCA \\
\hline
\end{tabular}



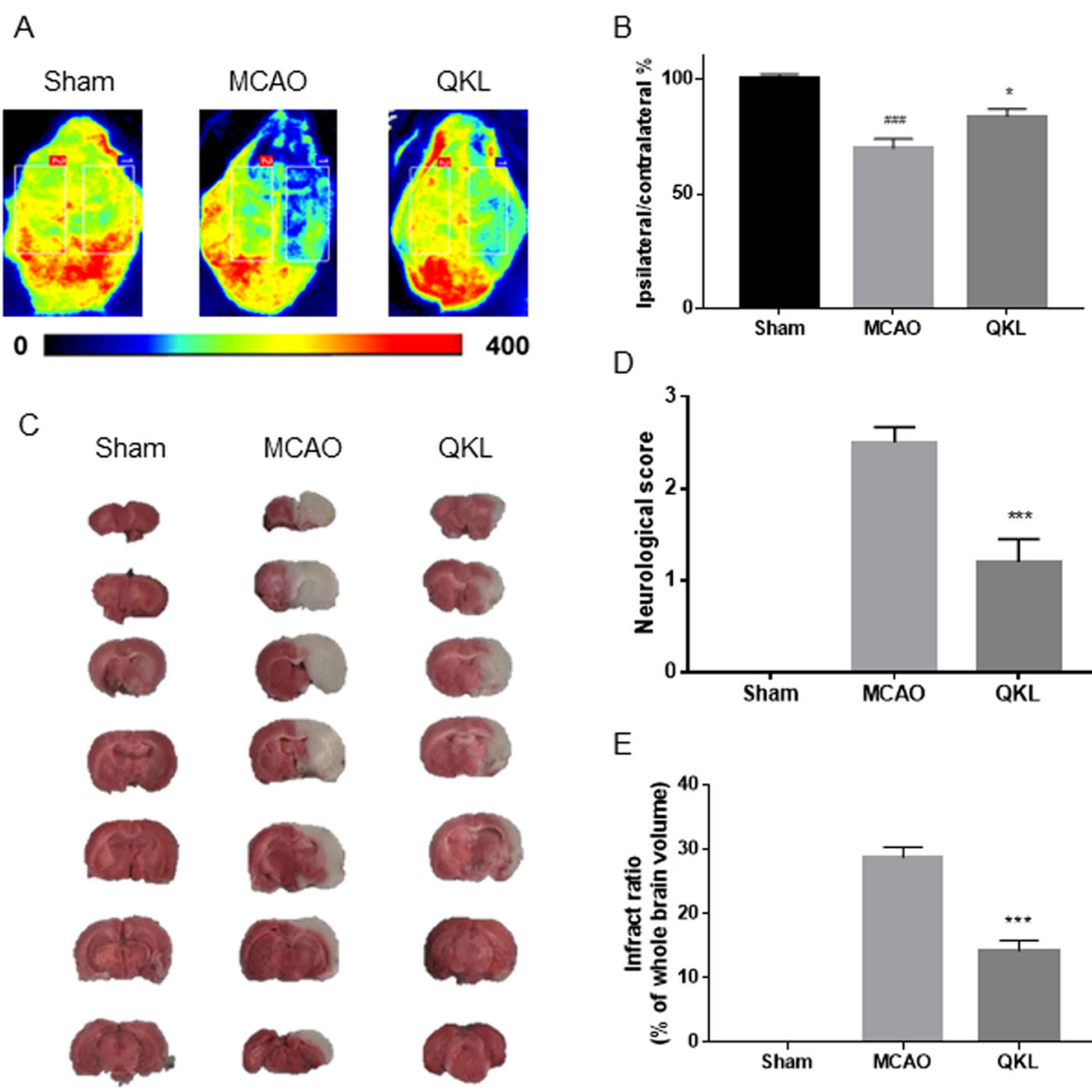

D
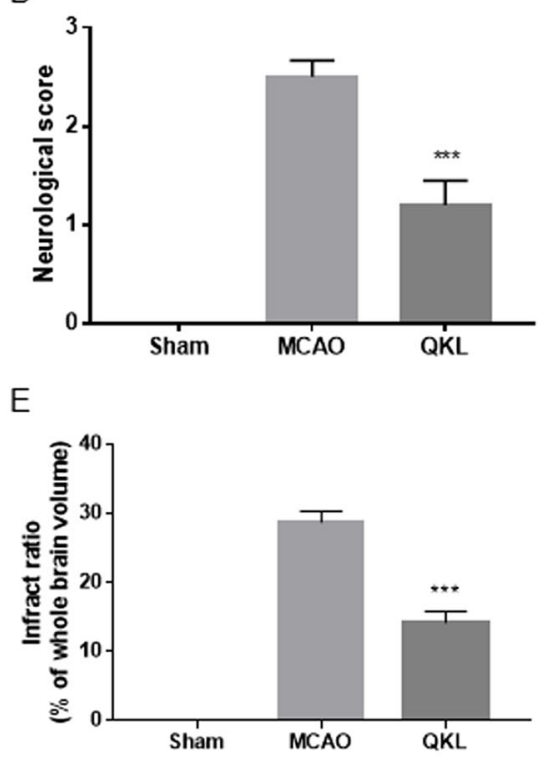

Fig. 1 Effects of Qingkailing (QKL) injection on ischemic injury in vivo. a Effects of QKL on region cerebral blood flow. b Quantitative analysis of regional cerebral blood flow. c Representative pictures of brain sections stained with $2 \%$ TTC. d Effect of QKL on neurological deficit scores. e Quantitative analysis of cerebral infarct volume. Data points indicate means \pm SEM from 10 individual rats in each group. Vs Sham group, $\# \# \#<0.001$, Vs MCAO group, ${ }^{*} p<0.05,{ }^{* * *} p<0.001$

shown in Fig. 5b-e, the level of NLRP3, ASC, Clcaspase-1, and Cl-IL-1 $\beta$ were increased in the MCAO group compared to the Sham group (Vs Sham, $p=0.023$, $p=0.0330, p=0.0010$ and $p=0.0001$, respectively). The levels of NLRP3, Cl-caspase-1, and Cl-IL-1 $\beta$ were significantly decreased after QKL treatment (Vs MCAO, $p=$ $0.0371, p=0.0165, p=0.0157$, respectively). The level of ASC was also increased after QKL treatment even though a significant difference was not observed (Vs MCAO, $p=0.1816)$. We used quantitative polymerase chain reaction (qPCR) to further evaluate the mRNA levels of NLRP3, ASC, caspase 1 , and IL-1 $\beta$. As shown in Fig. $5 f-i$, the level of NLRP3, Cl-caspase-1, and IL-1 $\beta$ mRNA were significantly reduced after QKL treatment (Vs MCAO, $p=0.0031, p=0.0017, p=0.0057$, respectively) and the level of ASC mRNA was reduced without significance (Vs MCAO, $p=0.3437$ ), which corresponded to the Western blot analysis result. We further investigated the activation of AMPK, a recently identified upstream protein of the NLRP3 inflammasome. The results indicated that QKL treatment activated AMPK via phosphorylation (Vs MCAO, $p=$ 0.0125) as shown in Fig. 6a-b.

\section{Effects of QKL on the oxidative stress}

An over accumulation of free radicals induced by cerebral ischemia-reperfusion injury can activate the NLRP3 inflammasome. SOD is an effective free-radical scavenger and an important antioxidant enzyme that scavenges oxygen free radicals after cerebral ischemic events. In Fig. 6c, our data show that QKL increased the level of SOD $(F(2,15)=7.702, p=0.005)$, which reduced the oxidative stress (Vs MCAO, $p=0.0053$ ). The level of MDA, an important lipid-peroxidation product, was also found significant different among these groups ( $F(2$, $15)=4.813, p=0.0001)$. In the ischemic brain, MDA was found to be downregulated after QKL treatment (Vs MCAO, $p=0.0015$ ), as shown in Fig. $6 \mathrm{~d}$. 
A
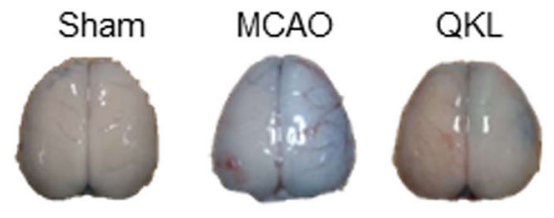

C
B

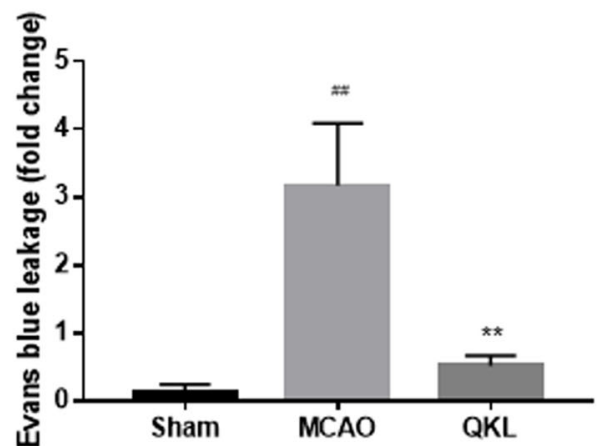

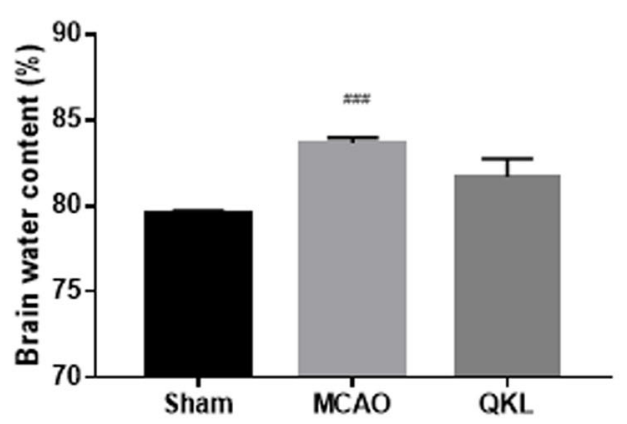

Fig. 2 Effects of QKL on post-stroke blood-brain barrier (BBB) dysfunction. a Representative pictures of Evans blue (EB) leakage analysis. $\mathbf{b} E B$ leakage analysis to evaluate prevention of BBB dysfunction by QKL. c Brain water content of each hemisphere. Data points indicate means \pm SEM

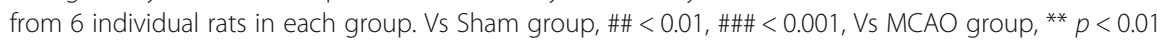

\section{Discussion}

Cerebral ischemia diminishes the oxygen supply, which damages the brain cells leading to neurological dysfunction and cerebral infarction. Challenges still exist in the management of cerebral ischemia, and more efficient agents to treat cerebral ischemia have yet to be identified. QKL is a famous CFDA approved Chinese medicine that has been clinically used throughout China for over 30 years. Understanding of biological mechanism of QKL from experimental studies may help developing potential clinical strategies of QKL to treat cerebral ischemia. The present study showed that QKL injection protected the brain against cerebral ischemia-reperfusion injury, including improved $\mathrm{rCBF}$, decreased neurological deficits and brain infarct volumes, inhibited BBB injuries and suppressed post-stroke inflammatory responses. Using Nissl staining and TUNEL analysis, we found that QKL suppressed brain cell apoptosis, which is the biological mechanism leading to the clinical symptoms of cerebral ischemia. In line with our study, other studies indicated that QKL injection improves neurological function, suppresses BBB dysfunction, and inhibits inflammatory responses [19, 25]. However, the underlying mechanism of the QKL anti- ischemic effect remains unknown. Baicalin, geniposide, cholic acid, hyodeoxycholic acid, chlorogenic acid and neochlorogenic acid were identified as six dominant ingredients absorbed into blood according to UPLC-MS/MS analysis [31]. These ingredients were reported to protect brain against cerebral ischemia.

Recent studies highlighted anti-inflammation effect of QKL injection. Our results indicated that QKL was able to modulate the NLRP3 inflammasome activation. Indeed, no matter whether the target is upstream or downstream in the NLRP3 inflammasome pathway at the molecular level, modulating the expression, assembly, activation and secretion of the NLRP3 inflammasome and IL- $1 \beta$ exerts a protective effect on cerebral ischemia-reperfusion injury. Better functional outcomes, decreased infarction volumes and oedema formations, preservation of BBB permeability, and reduced inflammatory pathology against cerebral ischemia-reperfusion injury was observed in a preclinical study [32]. QKL treatment inhibited the transcription of NLRP3, caspase 1 , and IL-1 $\beta$, which suppressed activation of NLRP3 inflammasome signalling pathway. Western blot analysis also indicated that expression of the NLRP3 protein was downregulated by QKL treatment. NLRP3 recruited 

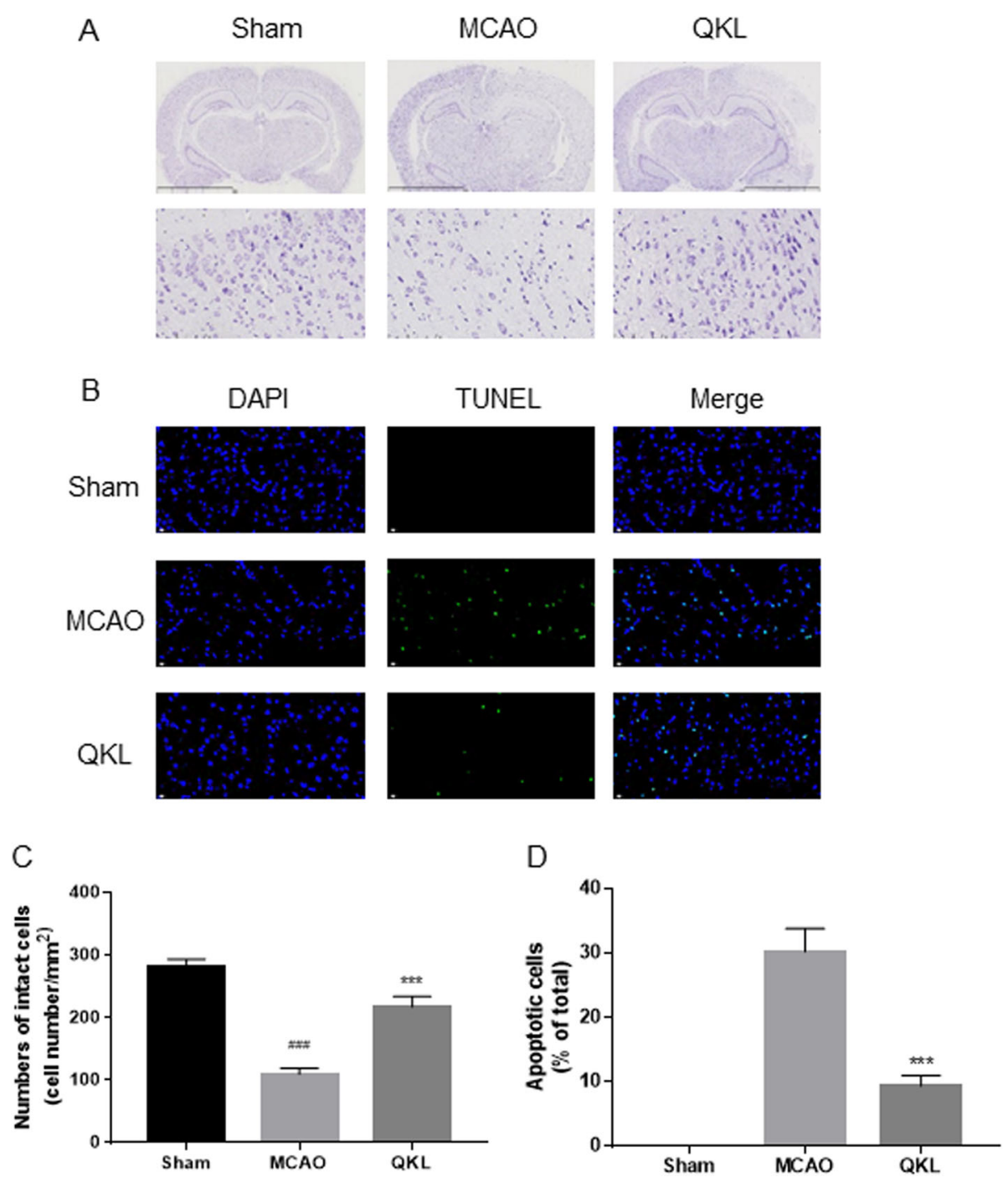

Fig. 3 Anti-apoptosis effects of QKL. a Representative pictures of Nissl staining and $\mathbf{b}$ representative images of cell apoptosis stained with the TUNEL method. c Relevant quantitative analysis of intact cell number and $\mathbf{d}$ rate of apoptotic cells in each group. Data points indicate means \pm SEM. Vs Sham group, \#\#\# $<0.001$, Vs MCAO group, ${ }^{* *} p<0.001$. At least three independent experiments were performed for each group

ASC, which in turn recruited caspase-1, causing its activation. Cellular pyroptosis was induced by activated caspase- 1 . And caspase- 1 also processed pro-IL-1 $\beta$ to a mature form, which was rapidly secreted into the extracellular matrix activating inflammatory responses. Therefore, activated caspase- 1 and IL- $1 \beta$ are two important molecules to evaluate the activation of the NLRP3 inflammasome. Our results showed that QKL reduced the level of $\mathrm{Cl}$-caspase- 1 and $\mathrm{Cl}-\mathrm{IL}-1 \beta$, which is to say that QKL was able to influence NLRP3 inflammasome activation through related protein expressions and functions of both molecules.

To further understand the underlying mechanism behind QKL in NLRP3 inflammasome activation, we tested AMPK activation, which was considered to be a target for modulating inflammation and oxidative stress in multiple pathophysiologic conditions [33, 34]. QKL treatment activated the phosphorylation of AMPK, improved the level of SOD, and decreased the level of MDA, indicating that QKL plays a role in AMPK signalling and oxidative stress. As mentioned previously, activation of the AMPK signal inhibits the NLRP3 inflammasome via multiple biological mechanisms. Blocking AMPK activation induced by molecular inhibitors, such as compound $\mathrm{C}$ or sunitinib, was associated with an over-active NLRP3 inflammasome signalling pathway in vivo and in vitro $[35,36]$. The direct interaction between AMPK and NLRP3 protein has not been reported to date. The most discussed mechanism is activated AMPK signal regulation of mitochondrial homeostasis, which is strongly connected to NLRP3 activation. Following mitochondrial 
A

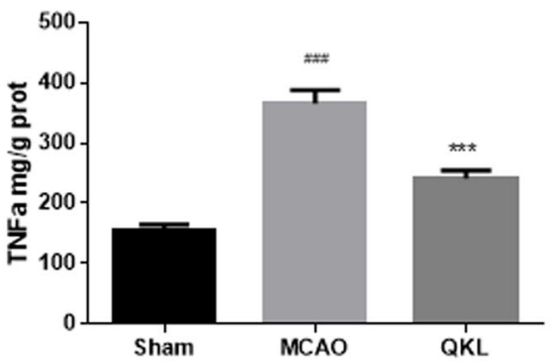

C

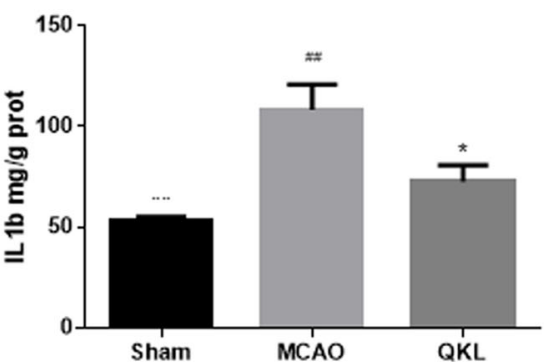

E

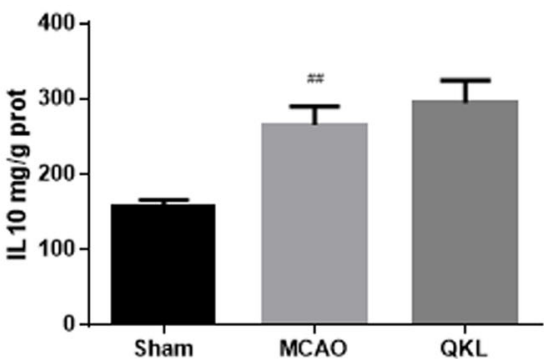

B

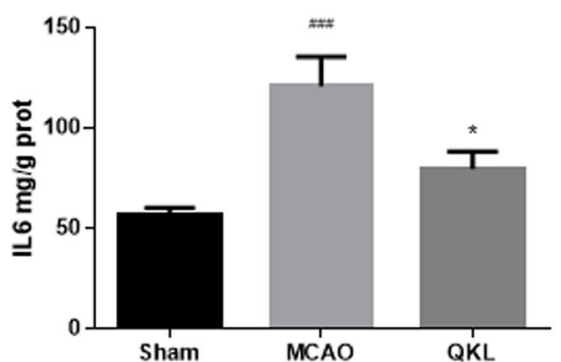

D

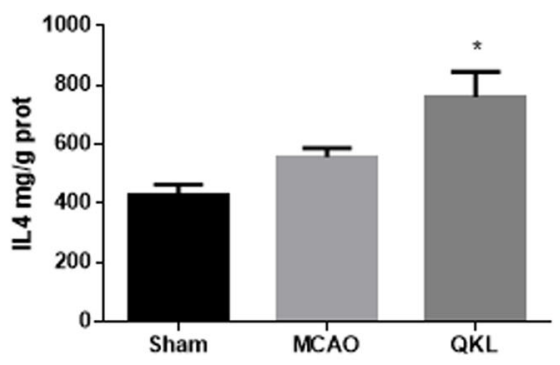

Fig. 4 Analysis of the expression of pro-inflammatory cytokines, TNF-a, IL-6 and IL-1 $\beta$, and anti-inflammatory cytokines, IL-4 and IL-10. a-c analysis of pro-inflammatory cytokines using enzyme-linked immunosorbent assay (ELISA) method for TNF-a, IL-6 and IL-1 $\beta$, respectively. $\mathbf{d}$-e analysis of anti-inflammatory cytokines using the ELISA method for IL-4 and IL-10, respectively. Data points indicate means \pm SEM. Vs Sham group, \#\#<0.01, $\# \# \#<0.001$, Vs MCAO group, ${ }^{*} p<0.05,{ }^{* *} p<0.001$. At least three independent experiments were performed for each group

dysfunction, AMPK is phosphorylated and activated to avoid risks of bioenergetic deficiencies. Furthermore, mitochondria are involved in many pathological processes as a consequence of their central role in reactive oxygen species (ROS) production [37]. AMPK is likely to be involved in cellular defenses against oxidative stress induced by mitochondrial ROS through increased SOD levels [38]. Previous research has indicated that high levels of ROS under various cellular stresses will activate the NLRP3 inflammasome signal pathway [39]. In detail, excess ROS induces the ROS scavenging protein thioredoxin from thioredoxin interacting/inhibiting protein (TXNIP), which binds to NLRP3 protein and modulates its assembly via oligomerization [40]. Revealing the functional interaction between AMPK and NLRP3 is important, as it demonstrates a novel relationship between inflammation/ROS and energy homeostasis.

The present research was the first preclinical study to examine the regulatory effect of $\mathrm{QKL}$ injection on AMPK/NLRP3 inflammasome pathway activation following cerebral ischemia-reperfusion injury. Actually, some ingredients in QKL injection have been considered to be critical regulators in the activation of AMPK/ NLRP3 inflammasome pathways, such as isoliquiritigenin [41], cholic acid [42] and baicalin [43, 44]. However, the direct interaction between the ingredients and the core protein in the AMPK/NLRP3 pathway remained unknown. Future studies should seek to identify the potential ingredients in the QKL injection that 


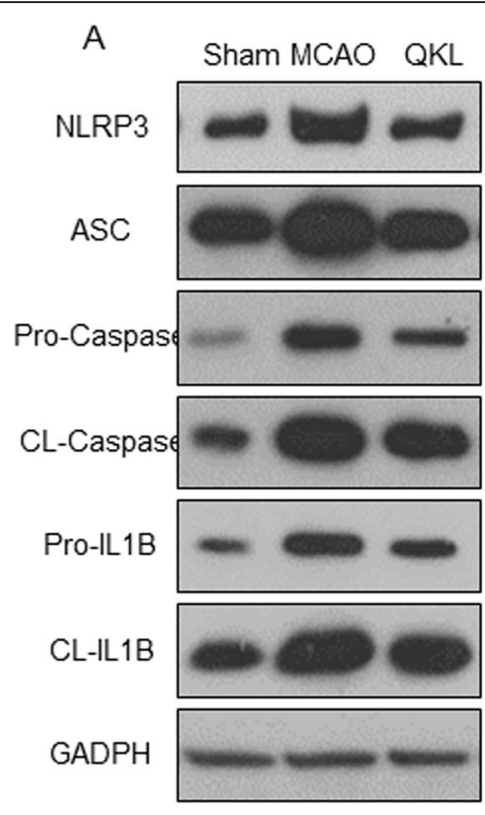

B
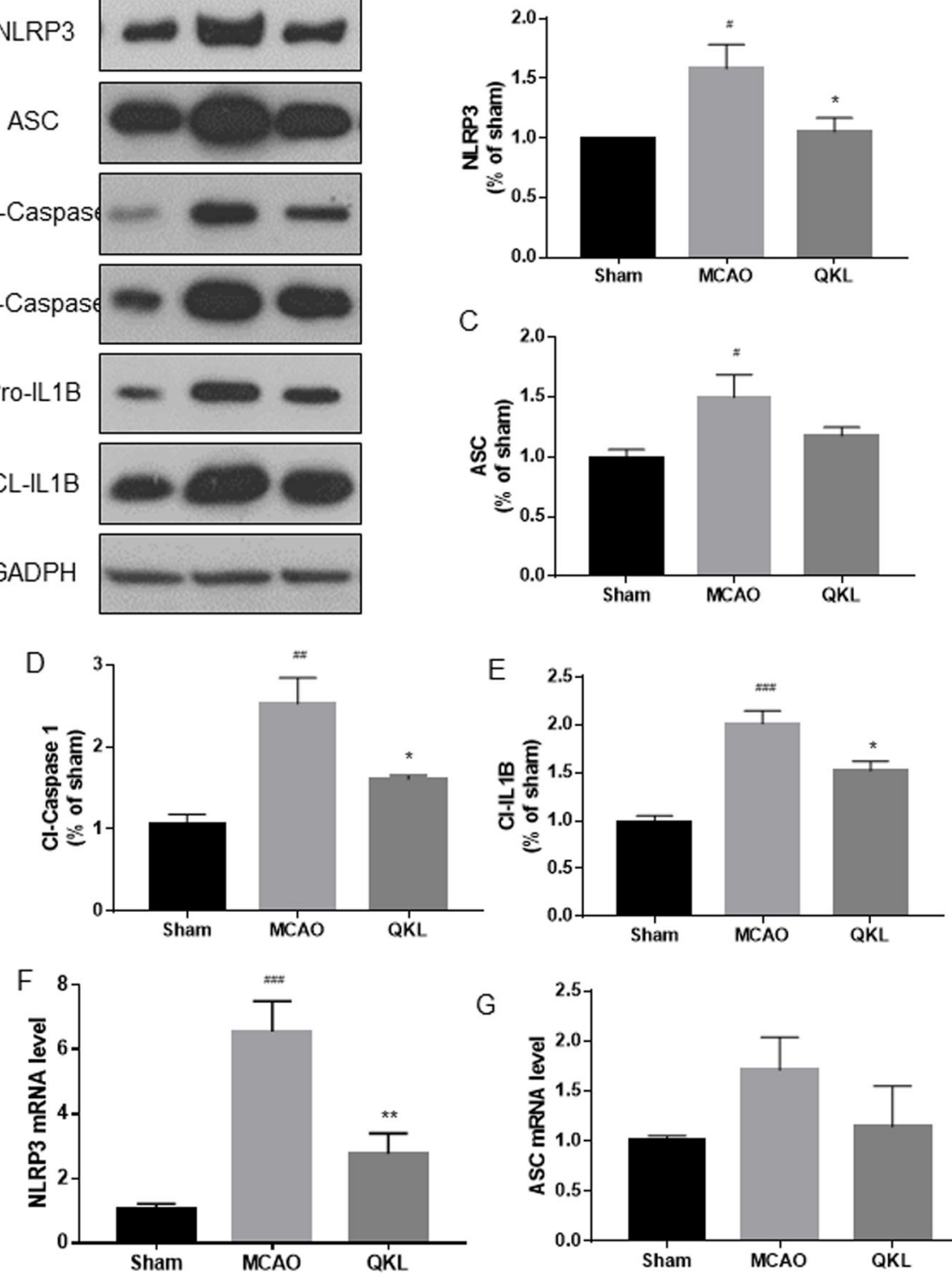

G
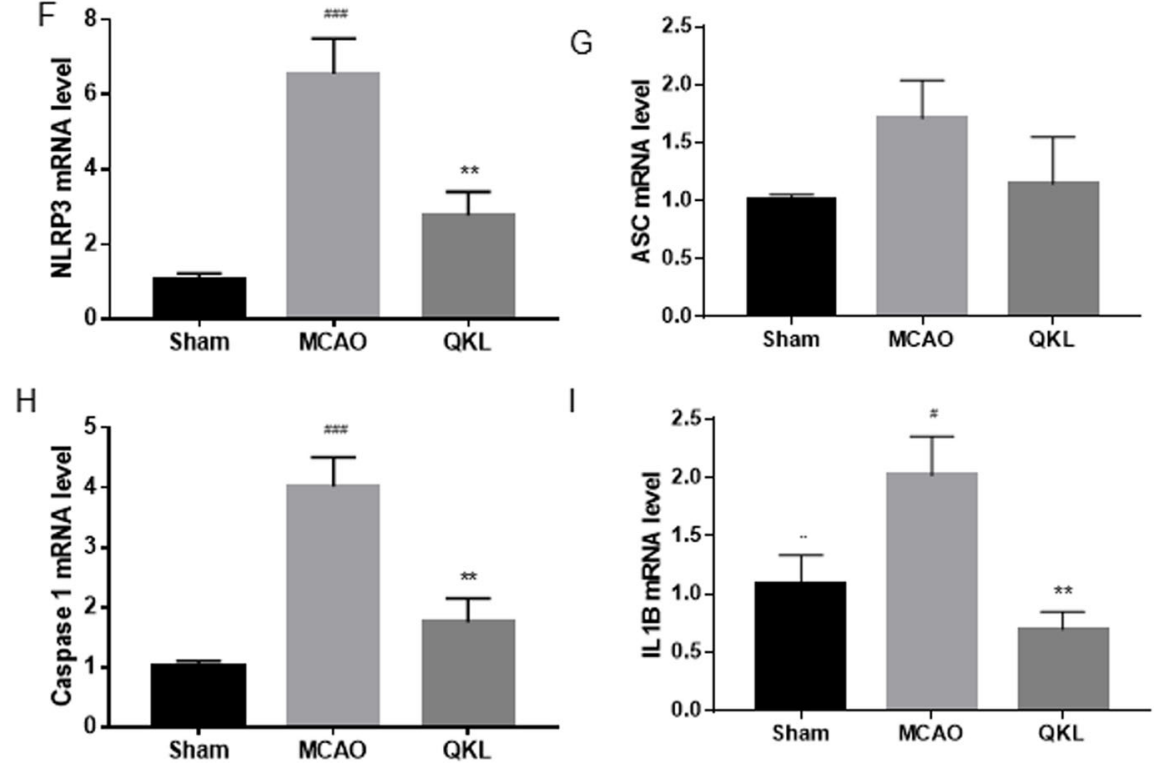

Fig. 5 QKL inhibited NLRP3 inflammasome activation post-stroke. (A) Western blot analysis of NLRP3, ASC, pro-caspase 1, cleaved-caspase 1, proIL-1 $\beta$, cleaved-IL-1 $\beta$ and GAPDH. (B-E) Quantitative analysis of NLRP3, ASC, Cl-caspase 1 and Cl-IL-1 $\beta$ expression. (F-I) Quantitative polymerase chain reaction (QPCR) analysis of NLRP3, ASC, caspase 1 and IL-1 $\beta$ mRNA. Data points indicate means \pm SEM. Vs Sham group, \#<0.05, \#\#<0.01, $\# \# \#<0.001$, Vs MCAO group, ${ }^{*} p<0.05$, ${ }^{* *} p<0.01$. At least three independent experiments were performed for each group 


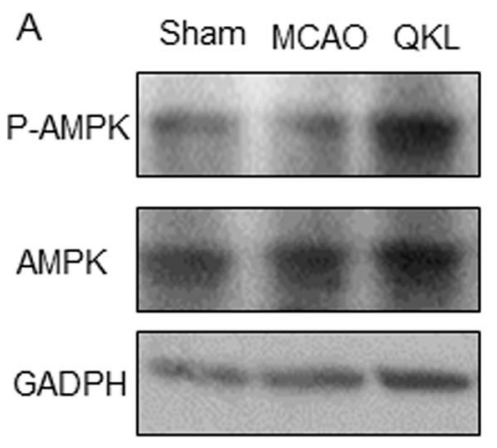

C

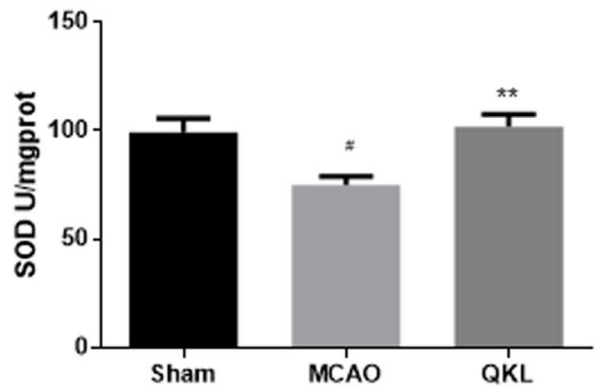

B

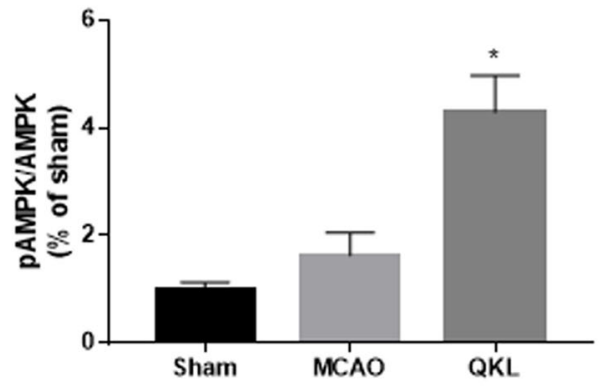

D

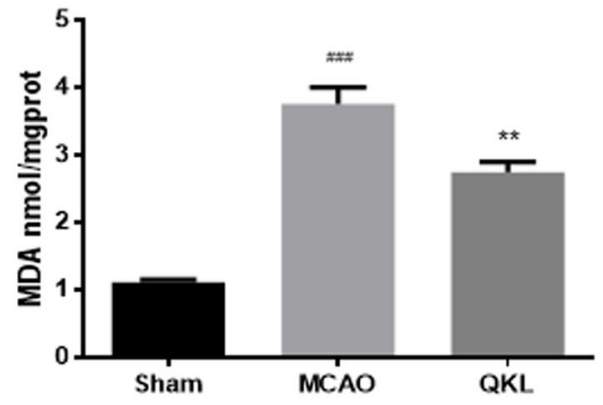

Fig. 6 QKL activated AMPK and decreased oxidative stress levels. a Western blot analysis of p-AMPK, AMPK and GAPDH. b Quantitative analysis of the p-AMPK: AMPK expression ratio. c Effect of QKL on the SOD level. d Effect of QKL on the MDA level. Data points indicate means \pm SEM. Vs Sham group, \#<0.05, \#\#\# 0.001, Vs MCAO group, ${ }^{*} p<0.05$, ${ }^{* *} p<0.01$. At least three independent experiments were performed for each group

target the AMPK/NLRP3 inflammasome signal pathway in the treatment of cerebral ischemia. The identification of the specific ingredient will facilitate the translation of basic science to the clinical setting.

\section{Conclusions}

In conclusion, this study elucidated that QKL relieved cerebral ischemia reperfusion injury and suppressed inflammatory response, at least in part, by inhibiting AMPK-mediated the activation of NLRP3 inflammasome. Therefore, our data might supply a new insight into the potential of QKL injection in the treatment of cerebral ischemia and help to understand the role of QKL injection on immune system in the brain.

\begin{abstract}
Abbreviations
AMPK: AMP-activated protein kinase; ASC: Apoptosis-associated speck-like protein containing a CARD; BBB: Blood brain barrier; CNS: Central nervous system; DAMPs: Damage-associated molecular patterns; NLRP3: Nucleotidebinding oligomerization domain (NOD)-like receptor (NLR) Pyrin domain containing 3; PAMPs: Pathogen-associated molecular patterns; PRRs: Pattern recognition receptors; QKL: Qingkailing; tMCAO: Transient MCAO
\end{abstract}

\section{Acknowledgments}

We thank Elsevier Language Services for providing language assistance and proofreading the manuscript. This work was supported by the Classical Prescription Basic Research Team of the Beijing University of Chinese Medicine.

\section{Authors' contributions}

FFC, XQW, and QGW designed this study, and CYM participated in the study design and wrote the article. TX, XY, SZ, SLL, and YSG contributed to animal experiment. SNF and CXL helped analyze the data and CMZ and XQW created the illustrations. All authors read and approved the final manuscript.

\section{Funding}

This study was financially supported by the National Natural Science Foundation of China (81430102, 81774122, 81473789, 81774030, 81373886 and 81303260 ). These funding bodies provided important supports during design of the study and collection, analysis, and interpretation of data.

\section{Availability of data and materials}

The supporting materials can be obtained upon request via email to the corresponding author.

\section{Ethics approval and consent to participate}

The animal experimental design and protocols used in this study were approved by the Ethics Review Committee for Animal Experimentation at the Beijing University of Chinese Medicine. All the experimental procedures were performed in accordance with the Regulations for the Administration of Affairs Concerning Experimental Animals approved by the State Council of People's Republic of China.

\section{Consent for publication}

Not applicable.

\section{Competing interests}

The authors declare that they have no competing interests.

\section{Author details}

${ }^{1}$ School of Traditional Chinese Medicine, Capital Medical University, 10 Youanmenwai, Xitoutiao, Fengtai District, Beijing 100069, China. ${ }^{2}$ School of Traditional Chinese Medicine Department, Beijing University of Chinese 
Medicine, 11 Beisanhuandong Road, Chao Yang District, Beijing 100029, China.

\section{Received: 28 August 2018 Accepted: 9 October 2019} Published online: 20 November 2019

\section{References}

1. Feigin $\mathrm{VL}$, Krishnamurthi RV, Parmar P, Norrving B, Mensah GA, Bennett DA, Barker-Collo S, Moran AE, Sacco RL, Truelsen T. Update on the global burden of ischemic and hemorrhagic stroke in 1990-2013: the GBD 2013 study. Neuroepidemiology. 2015;45(3):161-76.

2. Benjamin EJ, Blaha MJ, Chiuve SE, Cushman M, Das SR, Deo R, Floyd J, Fornage M, Gillespie C, Isasi C. Heart disease and stroke statistics-2017 update: a report from the American Heart Association. Circulation. 2017; 135(10):e146-603.

3. Powers W, Rabinstein A, Ackerson T, Adeoye O, Bambakidis N, Becker K, Biller J, Brown M, Demaerschalk B, Hoh B. 2018 guidelines for the early management of patients with acute ischemic stroke. Stroke. 2018;49(3):e46e97.

4. Huang J, Wang T, Yu D, Fang X, Fan H, Liu Q, Yi G, Yi X, Liu Q. IHomocarnosine attenuates inflammation in cerebral ischemia-reperfusion injury through inhibition of nod-like receptor protein 3 inflammasome. Int J Biol Macromol. 2018;118:357-64.

5. Cai W, Zhang K, Li P, Zhu L, Xu J, Yang B, Hu X, Lu Z, Chen J. Dysfunction of the neurovascular unit in ischemic stroke and neurodegenerative diseases: an aging effect. Ageing Res Rev. 2017:34:77-87.

6. Hoseini Z, Sepahvand F, Rashidi B, Sahebkar A, Masoudifar A, Mirzaei H. NLRP3 inflammasome: its regulation and involvement in atherosclerosis. J Cell Physiol. 2018;233(3):2116-32.

7. Elliott El, Sutterwala FS. Initiation and perpetuation of NLRP3 inflammasome activation and assembly. Immunol Rev. 2015;265(1):35-52.

8. Kanneganti T-D. Inflammatory bowel disease and the NLRP3 Inflammasome. N Engl J Med. 2017;377(7):694-6.

9. Fann DY-W, Lee S, Manzanero S, Tang S, Gelderblom M, Chunduri P, Bernreuther C, Glatzel M, Cheng Y, Thundyil J. Intravenous immunoglobulin suppresses NLRP1 and NLRP3 inflammasome-mediated neuronal death in ischemic stroke. Cell Death Dis. 2013;4(9):e790.

10. Dong Y, Fan C, Hu W, Jiang S, Ma Z, Yan X, Deng C, Di S, Xin Z, Wu G. Melatonin attenuated early brain injury induced by subarachnoid hemorrhage via regulating NLRP3 inflammasome and apoptosis signaling. J Pineal Res. 2016;60(3):253-62

11. Yang F, Wang Z, Wei X, Han H, Meng X, Zhang Y, Shi W, Li F, Xin T, Pang Q. NLRP3 deficiency ameliorates neurovascular damage in experimental ischemic stroke. J Cereb Blood Flow Metab. 2014:34(4):660-7.

12. Day E A, Ford R J, Steinberg G R. AMPK as a therapeutic target for treating metabolic diseases[J]. Trends in Endocrinol Metab. 2017;28(8):545-60.

13. Cordero MD, Williams MR, Ryffel B. AMP-activated protein kinase regulation of the NLRP3 inflammasome during aging[J]. Trends inEndocrinol Metab. 2018;29(1):8-17.

14. Shen P, Hou S, Zhu M, Zhao M, Ouyang Y, Feng J. Cortical spreading depression preconditioning mediates neuroprotection against ischemic stroke byinducing AMP-activated protein kinase-dependent autophagy in a rat cerebral ischemic/reperfusion injury model. J Neurochem. 2017;140(5): 799-813.

15. Medicine BUoC: The study of novel dosage form of an Gong Niu Huang wan. J New Med 1975, 8(12):12-13.

16. Sun Liangming CF, Xuegian W, Qingguo W. A systematic review and Meta -analysis of Qingkailing injection on acute stroke. Zhongguo Zhongyi Jizheng. 2016;25(5):772-6.

17. Cheng F, Wang X, Lu Y, Zhong X, Zhao Y, Wang Q. Chinese medicine injection qingkailing for treatment of acute ischemia stroke: a systematicreview of randomized controlled trials[J]. Evid Based Complement Alternat Med. 2012;2012, Article ID 213172:7.

18. Wang $G$, Xie $Y$, Wang $L$, Wang $Q$, Jia $P$, Feng $B$. Analysis of drug combination characteristics of Qingkailing injection for treating abnormal inflammatory factors in real world. China J Chinese Materia Medica. 2017; 42(10):1984-90.

19. Mana L, Wang S, Zhu H, Xing Y, Lou L, Wu A, Dong B, Sun Y, Yang S, Wang L. Qingkailing Suppresses the Activation of BV2 Microglial Cells by Inhibiting Hypoxia/Reoxygenation-Induced Inflammatory Responses. Evidence-Based Complementray Alternative Med. 2014;2014(1):696218.
20. Zheng Y, Hou J, Liu J, Yao M, Li L, Zhang B, Zhu H, Wang Z. Inhibition of autophagy contributes to melatonin-mediated neuroprotection against transient focal cerebral ischemia in rats. J Pharmacol Sci. 2014;124(3):354-64.

21. Longa EZ, Weinstein PR, Carlson S, Cummins R. Reversible middle cerebral artery occlusion without craniectomy in rats. Stroke. 1989;20(1): 84-91.

22. Beker MC, Caglayan AB, Kelestemur T, Caglayan B, Yalcin E, Yulug B, Kilic U, Hermann DM, Kilic E. Effects of normobaric oxygen and melatonin on reperfusion injury: role of cerebral microcirculation. Oncotarget. 2015;6(31): 30604-14.

23. Wang W, Ma X, Han J, Zhou M, Ren H, Pan Q, Zheng C, Zheng Q. Neuroprotective effect of Scutellarin on ischemic cerebral injury by Downregulating the expression of angiotensin-converting enzyme and AT1 receptor. PLoS One. 2016;11(1):e0146197.

24. Chen B, Wang G, Li W, Liu W, Lin R, Tao J, Jiang M, Chen L, Wang Y. Memantine attenuates cell apoptosis by suppressing the Calpain-Caspase-3 pathway in an experimental model of ischemic stroke. Exp Cell Res. 2017; 351(2):163-72

25. Cheng F, Zhong X, Lu Y, Wang X, Song W, Guo S, Wang X, Liu D, Wang Q. Refined Qingkailing protects MCAO mice from endoplasmic reticulum stress-induced apoptosis with a broad time window. Evid Based Complement Alternat Med. 2012;2012 Article ID567872:12.

26. Poinsatte K, Selvaraj UM, Ortega SB, Plautz EJ, Kong X, Gidday JM, Stowe AM. Quantification of neurovascular protection following repetitive hypoxic preconditioning and transient middle cerebral artery occlusion in mice. J Vis Exp Jove. 2015;379(99):e52675.

27. Oh TW, Park KH, Jung HW, Park YK. Neuroprotective effect of the hairy root extract of Angelica gigas NAKAI on transient focal cerebral ischemia in rats through the regulation of angiogenesis. BMC Complement Altern Med. 2015:15(1):1-11.

28. Liu MW, Wei R, Su MX, Li H, Fang TW, Zhang W. Effects of Panax notoginseng saponins on severe acute pancreatitis through the regulation of mTOR/Akt and caspase-3 signaling pathway by upregulating miR-181b expression in rats. BMC Complement Altern Med. 2018;18(1):51.

29. Xu S, Zhong A, Ma H, Li D, Hu Y, Xu Y, Zhang J. Neuroprotective effect of salvianolic acid $B$ against cerebral ischemic injury in rats via the CD40/NF-1 ${ }^{\circ} \mathrm{B}$ pathway associated with suppression of platelets activation and neuroinflammation. Brain Res. 2017:1661, 37-48.

30. Cheng F, Ma C, Wang X, Zhai C, Wang G, Xu X, Mu J, Li C, Wang Z, Zhang $X$. Effect of traditional Chinese medicine formula Sinisan on chronic restraint stress-induced nonalcoholic fatty liver disease: a rat study. BMC Complement Alternat Med. 2017;17(1):203.

31. Zhang Z, Qin L, Peng L, Zhang Q, Wang Q, Lu Z, Song Y, Gao X. Pharmacokinetic-pharmacodynamic modeling to study the antipyretic effect of Qingkailing injection on pyrexia model rats. Molecules. 2016; 21(3):317

32. Ismael S, Zhao L, Nasoohi S, Ishrat T. Inhibition of the NLRP3-inflammasome as a potential approach for neuroprotection after stroke. Sci Rep. 2018;8(1): 5971.

33. Kim MJ, Kim EH, Pun NT, Chang J-H, Kim J, Jeong J-H, Choi DY, Kim S-H, Park P-H. Globular adiponectin inhibits lipopolysaccharide-primed inflammasomes activation in macrophages via autophagy induction: the critical role of AMPK signaling. Int J Mol Sci. 2017;18(6):1275.

34. Jiang S, Li T, Ji T, Yi W, Gu C. AMPK: potential therapeutic target for ischemic stroke. Theranostics. 2018;8(16):4535-51.

35. Bullón P, Alcocer-Gómez E, Carrión AM, Marín-Aguilar F, Garrido-Maraver J, Román-Malo L, Ruiz-Cabello J, Culic O, Ryffel B, Apetoh L. AMPK phosphorylation modulates pain by activation of NLRP3 Inflammasome. Antioxid Redox Signal. 2016;24(3):157.

36. Qiu J, Wang M, Zhang J, Cai Q, Lu D, Li Y, Dong Y, Zhao T, Chen H. The neuroprotection of Sinomenine against ischemic stroke in mice by suppressing NLRP3 inflammasome via AMPK signaling. Int Immunopharmacol. 2016;40:492-500.

37. Nguyen H, Zarriello S, Rajani M, Tuazon J, Napoli E, Borlongan CV. Understanding the role of dysfunctional and healthy mitochondria in strokepathology and its treatment. Int J Mol Sci. 2018;19(7):2127.

38. Garrido-Maraver J, Paz MV, Cordero MD, Bautista-Lorite J, Oropesa-Ávila M, de la Mata M, Pavón AD, De LI, Alcocer-Gómez E, Galán F: Critical role of AMP-activated protein kinase in the balance between mitophagy and mitochondrial biogenesis in MELAS disease. Biochim Biophys Acta 2015, 1852(11):2535-2553. 
39. Heid ME, Keyel PA, Kamga C, Shiva S, Watkins SC, Salter RD. Mitochondrial reactive oxygen species induces NLRP3-dependent lysosomal damage and inflammasome activation. J Immunol. 2013;191(10):5230-8.

40. Yang SJ, Shao GF, Chen JL, Gong J. The NLRP3 inflammasome: an important driver of neuroinflammation in hemorrhagic stroke. Cell Mol Neurobiol. 2018;38(3):595-603.

41. Liu Q, Lv H, Wen Z, Ci X, Peng L. Isoliquiritigenin activates nuclear factor Erythroid-2 related factor 2 to suppress the NOD-like receptor protein 3 Inflammasome and inhibits the NF-KB pathway in macrophages and in acute lung injury. Front Immunol. 2017:8:1518.

42. Guo C, Xie S, Chi Z, Zhang J, Liu Y, Zhang L, Zheng M, Zhang X, Xia D, Ke Y. Bile acids control inflammation and metabolic disorder through inhibition of NLRP3 inflammasome. Immunity. 2016;45(4):802-16.

43. Li S, Sun X, Xu L, Sun R, Ma Z, Deng X, Liu B, Fu Q, Qu R, Ma S. Baicalin attenuates in vivo and in vitro hyperglycemia-exacerbated ischemia/ reperfusion injury by regulating mitochondrial function in a manner dependent on AMPK. Eur J Pharmacol. 2017:815:118-126.

44. Li CG, Yan L, Mai FY, Shi ZJ, Xu LH, Jing YY, Zha QB, Ouyang DY, He XH. Baicalin inhibits NOD-like receptor family, Pyrin containing domain 3 Inflammasome activation in murine macrophages by augmenting protein kinase a signaling. Front Immunol. 2017;8:1409.

\section{Publisher's Note}

Springer Nature remains neutral with regard to jurisdictional claims in published maps and institutional affiliations.

Ready to submit your research? Choose BMC and benefit from:

- fast, convenient online submission

- thorough peer review by experienced researchers in your field

- rapid publication on acceptance

- support for research data, including large and complex data types

- gold Open Access which fosters wider collaboration and increased citations

- maximum visibility for your research: over $100 \mathrm{M}$ website views per year

At BMC, research is always in progress.

Learn more biomedcentral.com/submissions 